\title{
ARTIFACT-CENTRIC SEMANTIC SOCIAL-COLLABORATIVE NETWORK IN AN ONLINE HEALTHCARE CONTEXT
}

\author{
Nazmul Hussain, Hai H Wang and Christopher D Buckingham \\ Engineering and Applied Science, Aston University, Birmingham, UK
}

\begin{abstract}
The emergence of Web 2.0 technology and associated social networking system, brings many possibilities and features for online collaboration. Several reference models, frameworks, tools and infrastructures have been proposed to support seamless interaction and communication between human entities in an online social environment. A few studies indicated that social networks are not only constructed on social connections of people, but also mediated by shared objects, known as object-centred sociality. However, most of the developed social software system was centred around the concept of maintaining human-centric social relationships only. This may be because of the common issues of exploiting social network approach for online collaboration related to maintaining the social behaviour, interaction and communication between human and artifacts themselves. These resources or artifacts (i.e., physical objects, software entities, documents, etc.) are active elements in a way that they may coordinate, cooperate, and even trigger collaborative work in a social environment, which is difficult understand and implement. Thus, it is essential to concentrate on exploring the artifact-centric social relations in a new generation of social-collaboration network.

This paper explores the concept and characteristics of the social software system and emphasise the importance and role of objects and artifact-centric sociality in a social-collaboration network. We also outline the benefits of semantic representation of social-collaborative network structure by extending existing social ontologies such as FOAF, SIOC, and DC that define additional concepts, properties and complex social relationship of humans, social objects and collaboration artifacts. Finally, we demonstrate the effectiveness of our proposed approach by applying it to a large-scale social-collaborative healthcare service called GRaCE-AGE within the United Kingdom.
\end{abstract}

\section{KEYWORDS}

Web 2.0, Social Network, Work Artifacts, Semantic Web, Ontology, Healthcare, GRaCE-Age

\section{INTRODUCTION}

The emergence of Web 2.0 and associated social media technologies such as wiki, blog, social network, etc. has dramatically changed the way to communicate and information sharing between people across the communities more interactively (Chen, Hendry, \& Huang, 2016). Social networking system, in particular, built on Web 2.0 technology that brings many possibilities and features for online collaboration, which is currently an active research topic in the area of social computing that attracted many computer scientists, academics and enterprise communities.

Collaboration in various communities is proven powerful for solving problems, building consensus, and helping in decision-making processes (Zaffar \& Ghazawneh, 2012). Traditionally, email is the most-widely used method for collaboration for its flexibility and ease of use in notifying and communicating with people. However, one of the drawbacks of exploitation of email is that people tend to use it for a variety of purposes and often in a quasi-synchronous manner (Abbattista et al., 2008). Thus, it is not truly real-time to meet the all collaboration and communication needs. On the other hand, traditional face-to-face community-engaged method is limited by geographic location, limited in resources, and/or uses one-way communications (Soto et al., 2016). In contrast, online collaboration tools are beneficial to overcome the limitations of specific kinds of collaboration activities such as time, distance and communication problems (Samuel, 2015). Some studies reveal that online collaboration processes are influenced by social and communication structures formed by the collaborators (Liptchinsky et al., 2014), and building relationships is fundamental to the success 
of collaborations (Bergstrom et al., 1995). Socially enabled collaboration empowers and encourages participation, conversation, openness, content creation and socialisation among a community of users (Gaál et al., 2015).

In these context, several reference models, frameworks, applications and infrastructures have been proposed to support seamless interaction and communication in a collaborative network environment. Sadly, most of these existing collaboration approaches and developed systems are mainly dedicated to people-centric for sharing common interests, goals, and areas of expertise (Zaffar \& Ghazawneh, 2012). For example, existing social software like Facebook, LinkedIn, Youtube, etc. is particularly dedicated to connecting friends and families, professional networking and media sharing (Arnaboldi et al., 2017). In contrast, few Web 2.0 based open social application (i.e., wikis, groupware, google docs, etc.) facilitates collaboration by providing a social context. Nevertheless, the collaborative objectives could be totally achieved using these applications. In fact, current approaches not paying much attention to artifact-centric relation, communication and collaboration in a context-based social network.

Object-centred sociality (Engeström, 2005) was discussed in the few literatures, whereby social structures and interactions can be centred around objects (Marie \& Gandon, 2011). It means that people do not just connect to each other, they also connect through shared objects and work artifacts (Begel \& Deline, 2009). For example, the relations between documents, a software component, a technical design and other work artifacts should represent various aspects and deliverables that may influence the collaboration process (Liptchinsky et al., 2014). However, one of the less focused areas is that existing collaboration modelling approaches do not clearly recognise how objects and artifacts establish and maintain social relationships, how they are used and understood by people, also how they tie people together around object-centric a social network (Nansen et al., 2014).

Moreover, without consistent structures and semantics, it is impossible to capture the social phenomenon of human and artifact-centric social interconnection. Promisingly, modelling of social-collaboration network can be enriched with Semantic Web's (Berners-Lee, Hendler, \& Lassila, 2001) ontological representation by linking concepts and properties together in the Web 2.0 era (Stan \& Villarceaux, 2009). In general, ontology explicitly specifies the concepts and properties (object properties and data properties), uses reasoning and inference mechanism to deduce new information through the rules (e.g., discover new relationships) (Oellinger \& Wennerberg, 2006). Additionally, recommendation services can take advantage of semantic reasoning capabilities to overcome common limitations of current systems and to achieve intelligent recommendation (Chen et al., 2016). Although, there are some well standard social ontologies (e.g., FOAF, SIOC, SKOS, DC) exists to semantically define social network structure, but they are not capable of represent the network of object or artifacts and their relationship with human entities.

The purpose of this paper is to explore the concept and characteristics of social software system and distinguish to what extent it can promote online collaboration. We discuss the importance and role of artifact-centric sociality in a social-collaboration network. Furthermore, we outline the benefits of semantic representation of object-centred social-collaborative network structure by extending existing social ontologies into a new ontology. Finally, we demonstrate the applicability of our proposed approach by applying it to a large-scale social-collaborative healthcare service called GRaCE-AGE (https://www.egrist.org/grace-age) within the United Kingdom that creates a canopy of care functionality by connecting older adults to care providers.

The paper is organized as follows: Section II describes background and motivation of work; Section III discuss some related work; Section IV presents the SSCN model; Section V proposes extended ontology; Section VI examines a case study with implementation; and Section VII discusses the findings and concludes with further research directions.

\section{BACKGROUND AND MOTIVATION}

This section presents the main motivation and overviews the most relevant theories, approaches and tools that can serve as the basis for the designing and implementing semantic social-collaboration network. 


\subsection{Motivation}

Online healthcare domain is a prime example of social enabled collaborative work where multiple people work together to provide care support to patients in hospital or at home (Xiao, 2005). Healthcare provision is a set of activities that demands the collaboration with each other (e.g., physicians, nurses, managers, patients, etc.) (Christopoulou et al., 2017) in order to improve the quality of patient's care, in terms of efficient research, cost decrease, good management of resources, etc. (Benhiba et al., 2017). Using social media tools, physicians can reach their patients easily to update them with a therapeutic plan, to answer disease-related questions, or simply to provide advice or reminders (Lapointe, Ramaprasad, \& Vedel, 2014). On the other hand, medical information system is mediated by artifacts like software modules and resources (i.e., hardware and human participants) that are usually spatially and functionally distributed (Christopoulou et al., 2017), which require a significant attention to define their social relationship and semantic representation in a collaborative environment. Thus, the motivation of this work is the development of a semantic social-collaborative network (SSCN) model, which will support communication and collaboration between care communities, patients and medical artifacts. Furthermore, semantic web technologies will improve interoperability between people and resources in healthcare practice through the utilisation of shared ontology.

\subsection{Web 2.0 and Social Software}

The advent and great success of a new generation of web-based and community-centred applications often defined under the label of social software (Lohmann et al., 2009). The core idea of social software is often characterise as a subset of the Web 2.0, and its application for supporting collaboration in organizations termed as "Enterprise 2.0" (Koch, 2008).

Collaboration is the process of working together of people, groups and organizations in a sociable environment to systematically solve a problem that could not be solved by an individual alone (Bergstrom et al., 1995). Previous research works indicate that social media tools like wikis are increasingly becoming popular for managing knowledge and collaboration within enterprises (Zaffar \& Ghazawneh, 2012). In general, social networking is extremely popular on the Internet, that utilises Web 2.0 features (Lima et al., 2014), which allow quick and easy communication methods between users for personal or professional purposes (Dey et al., 2018).

A social network is a social graph composed of users and other social entities such as multimedia contents, human, posts, places, events, etc. called nodes, which are interrelated or connected through common social relationships (e.g., friendship, following, likes, shares, etc.) either directly or indirectly called edges (Ghafoor \& Niazi, 2016). Similarly, advanced social network can be represented as graphs with nodes both for people and their work artifacts (e.g. shared document, workflow, email, etc.) that coordinate collaborative tasks (Begel \& Deline, 2009).

\subsubsection{Objects and Artifacts in Social Network}

Object-centred sociality is a new concept introduced by Jyri Engeström (Engeström, 2005) that examine how the inclusion of shared objects can enhance online social networking formed between people and shared objects (Marie \& Gandon, 2011). It describes the fact that strong social relationships are built mainly when individuals are grouped together around a shared object, and it mediates the ties between them (Kaldoudi et al., 2011).

\subsubsection{Semantic Social Network}

The Semantic web is an extension of the current web, in which information is given defined meaning, enabling machines and users to cooperate (Berners-Lee et al., 2001). An ontology is a semantic web technology that formally and explicitly specifies entities, attributes and properties related to a domain (Obeid et al., 2018). In the conceptual model, entities are defined as Classes a collection of instances, Properties represent relations between classes, and Instances also called objects or individuals are concrete members of a class.

Few researchers explored that social networks would benefit from utilising existing social ontologies such as FOAF, SIOC, SKOS, and DC ontology (Erétéo et al., 2011; Limpens et al., 2008; Passant, 2007). FOAF (Friend-Of-A-friend)(http://www.foaf-project.org/) is the most popular ontology used for describing people, their relationships and social activity, SIOC (Semantically-Interlinked Online Communities) (http://sioc-project.org) describes the most common elements present on Web of communities, SKOS (Simple Knowledge Organization System) ontology (https://www.w3.org/2004/02/skos/) describes systems of organization of knowledge, and DC (Dublin Core) metadata vocabularies (http://dublincore.org/) can be used 
to describe digital resources (video, images, web pages, etc.), as well as physical resources such as books or CDs, and objects like artworks. However, these ontologies are not capable to fulfill the all requirement of a domain-specific context-aware social network structure.

\section{RELATED WORK}

There are various definitions, characteristics and tools for online collaboration have been proposed that highlight how to use Web 2.0 technology to support knowledge sharing, communication and collaboration in organisations. (Exter et al., 2012) explores the use of Web 2.0 technologies for collaborative learning in a higher-education context, including a review of the key strengths and weaknesses of Web 2.0 technology was introduced. (Arif, 2017) highlighted the new online collaboration tools like as Slack, Trello, or Asana, can serve as a means for focused communication and project collaboration both inside and outside the academia. Previously, Wikipedia was a well-known online collaborative platform that attracted millions of Internet users from all over the world, which enables users to view, create, edit, or remove articles on different topics (Zaffar \& Ghazawneh, 2012). (Murthy \& Lewis, 2015) cited that the social aspects could help build trust in virtual teams, and this trust could provide an important foundation for collaboration. (Storey et al., 2014) presents a review of research that examines the past, present, and future roles of social media channels in software engineering from 1968 to the present day. IBM describes their social software called "Lotus Live" (https://www.ibm.com/developerworks/lotus/), which is a comprehensive portfolio of these state-of-the-art collaboration tools that are applicable to organizations of all sizes, simplify and improve daily business interactions with customers, partners and colleagues. (Thelwall \& Kousha, 2015) investigates ResearchGate, a social network site for academics to create their own profiles, list their publications and interact with others. (Neumann et al., 2005) discussed the systematic overview of communication and collaboration features of semantic social networking portals and a compared the methods for community discussion, and user-to-user links. Moreover, (Obeid et al., 2018) proposes the use of the ontology-based recommendation system to assist students in finding and selecting the relevant university and major subjects to their field of interest.

In contrast, (Nansen et al., 2014) argued that objects are social that play role in mediating human social relationships, and these objects have a social life of their own through the fact of their emergence, persistence, relations and death. In similar context, (Xiao, 2005) review field studies in healthcare and other domains on the role of artifacts in collaborative work and draw implications in three areas, including methodological, theoretical, and technological. Instead, (Maamar, Buregio, \& Sellami, 2015) discusses how to design and develop collaborative enterprise applications using business and social artifacts.

\section{THE PROPOSED SEMANTIC SOCIAL-COLLABORATION NETWORK MODEL}

The proposed Semantic Social-Collaborative Network (SSCN) is a general web application model with ontology support that can be used to implement context-aware collaborative network, where all kinds of participants, including human and non-human will work together in a social environment and possibly distributed.

\subsection{The Conceptual Model of the Framework}

To illustrate the system, a conceptual model of SSCN has been proposed, which is comprised of three core subsystems: (i) Social-Collaborative Network (SCN), (ii) Recommender System (RS), and (iii) Ontology Service Module (OSR) that all together forming a whole SSCN as shown in Figure 1.

Social-Collaborative Network (SCN) is a subsystem of SSCN and comprises of Social Network (SN) environment and Human Agents. SN maintains all kinds of social entities, properties and their interactions, communications and relationships. The Human Agents are physical existence of persons that create and manage their social profile and maintain social connections like friendship, membership, sharing, follows, etc., and interact with social objects and collaboration artifacts. These artifacts are kind of non-human entities, which can have social existence and relations in the $\mathbf{S N}$ environment and maintained by human. 

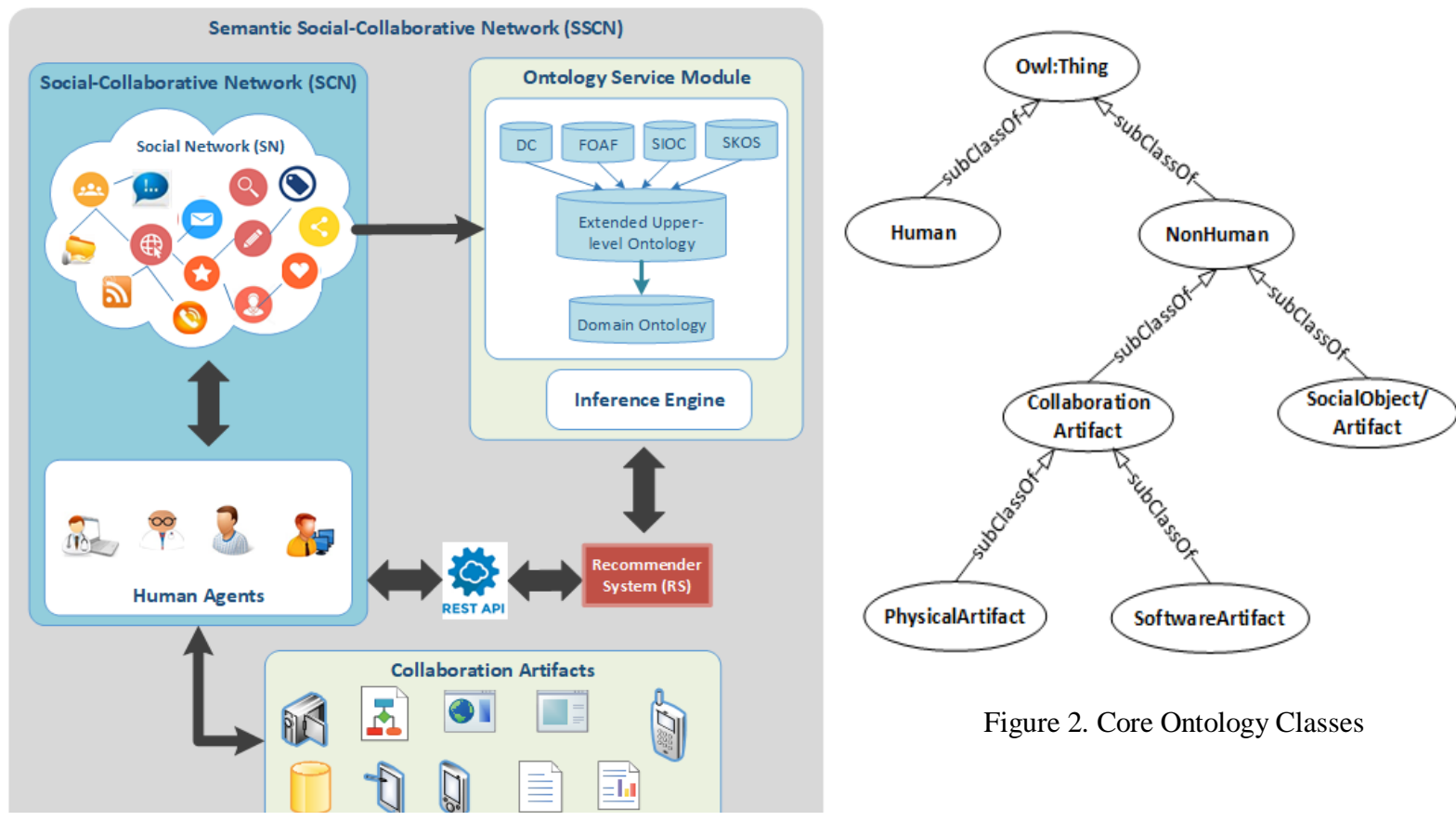

Figure 2. Core Ontology Classes

Figure 1. Semantic Social-Collaboration Network Model

Ontology Service Module (OSM) provides ontology management and inference service to the system, where extended upper-level ontology and domain-specific ontology is linked with existing FOAF, SIOC, SKOS and DC. This ontology integration formally defines the whole SCN structure, concepts and properties, and updates the ontology as changes happened in the network. The Recommender System (RS) is an integrated third-party software module that can provide functionality to generate personalised or collaborative recommendations based upon the ontology inference and semantic similarity of user profile, preferences, annotations, tags and interests. The RS can communicate and send and receive data from the $\mathbf{S C N}$ by a standard RESTful API. In this work, we do not focus on the low-level functionality and the development of internal logic of the recommendation system.

\section{EXTENDED ONTOLOGY MODELLING}

This extended ontology model discusses a way to represent artifact-centric social-collaboration network data in a shared and machine-readable way. Its main objective is to capture additional non-human related concepts, properties and relation in the social-collaborative network while reducing the unintended modeling costs and human efforts.

For the ease of development, we split our ontology model into a Core Ontology (CO), an extended upper-level Social-Collaboration Ontology (SCO) and a Domain Ontology (DO) for the flexible interface and extensibility for any domain-specific system implementation.

\subsection{Defining the "CoreOnto" Ontology}

In this section, we present a new "CoreOnto" ontology as shown in Figure 2 by defining a set of abstract concepts as the main building blocks for the representation of artifact-centric sociality discussed in this paper. It is a light-weight ontology that consisting of minimal core concepts and relationships about human and non-human entities, user-generated social objects and collaborative artifacts that may be further conceptualise into physical artifacts and software artifacts. 


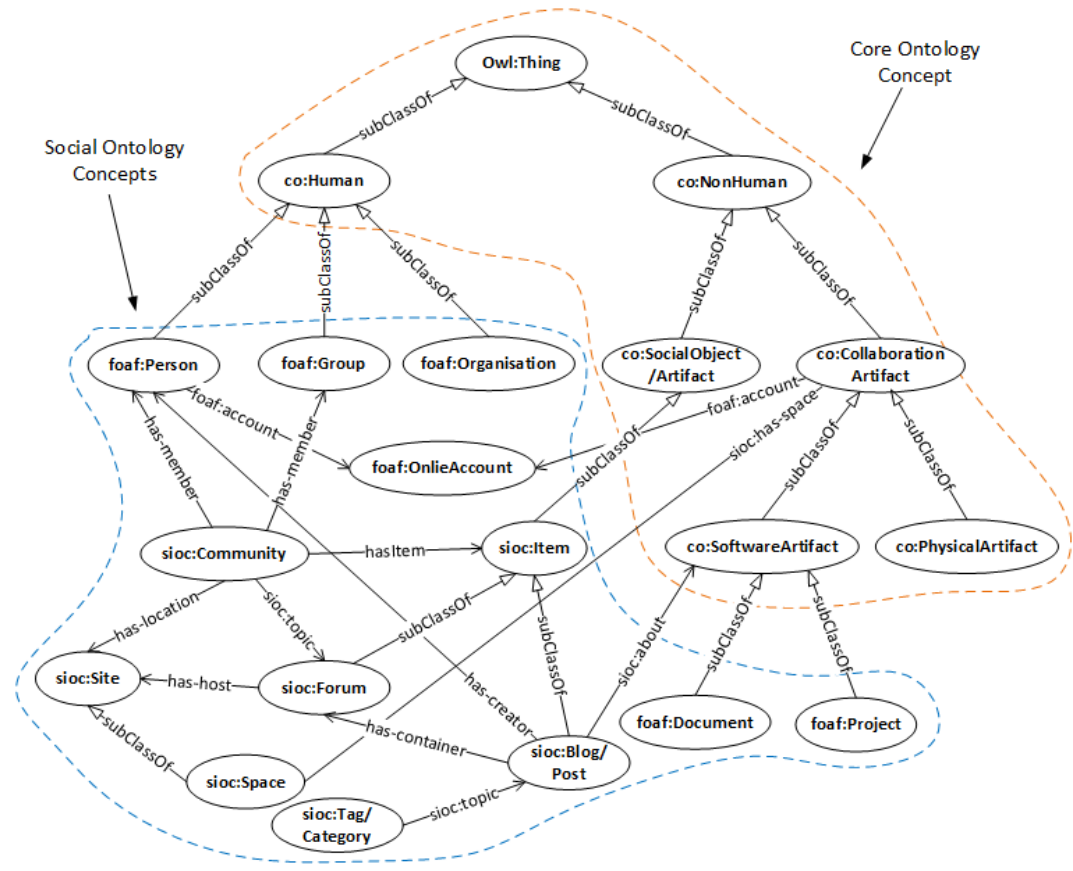

Figure 3. Extended Social-Collaboration Ontology

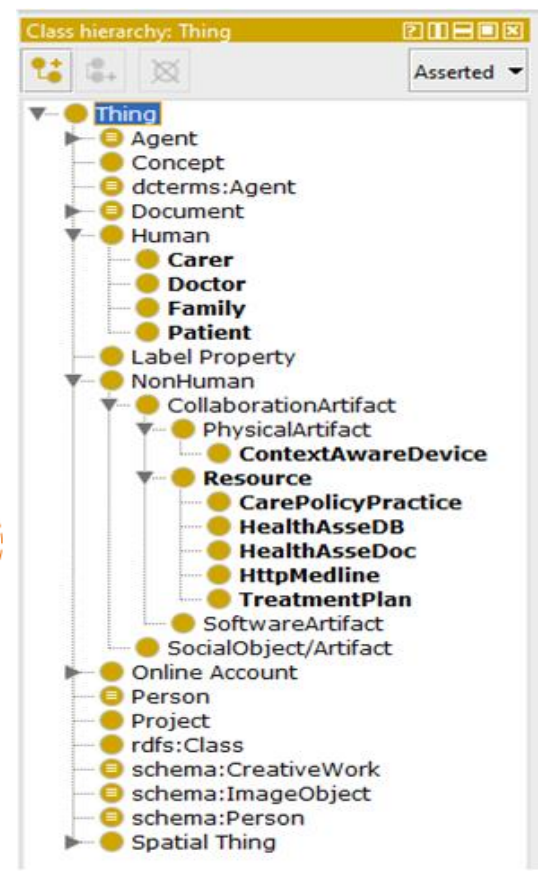

Figure 4. Ontology Implementation

\subsection{Upper-level "SocialCollaborationOntology"}

The extended upper-level "SocialCollaborationOntology", reuse the concepts and properties from "CoreOnto" ontology as well as existing FOAF and SIOC ontology as shown in Figure 3. This upper-level ontology is limited to very abstract conceptualisation that can be shared across domain-level ontology. In this ontology extension, we have reused few concepts and properties to interlink both ontologies together based on our case requirements. However, the domain-level ontology may inherit additional concepts and properties from the upper-level ontology or may define new terms and properties according to a domain-specific requirement.

\section{USE CASE: GRaCE-AGE HEALTHCARE SYSTEM}

In order to demonstrate the applicability of the proposed approach, we considered GRaCE-AGE (Galatean Risk and Care Environment) (https://www.egrist.org/grace-age) system, a web-based software system providing expertise directly connecting to the older adults and their network of carer's such as family, friends, clinical services to ensure they are safe, secure, and thriving (Buckingham et al., 2015). However, GRaCE-Age and many other similar online healthcare systems do not have complete control of sociality and not focusing on non-human participant (e.g., care artifacts and resources) and their interactions and communication, nor they are semantically described.

We believe that our proposed approach would fulfills this gap by implementing an extended social-collaborative care network of GRaCE-Age system and its relevant extended ontology, which will semantically represent the additional concepts, properties and their relationships. It will make social links between resources, individuals and community of practice in the social healthcare environment, which may improve care service and increased transparency throughout the patient's care journey and reduce healthcare costs. Additionally, the integrated recommendation service may suggest new treatment plan based on the agumented post, blogs, or assessment data, or suggest new contacts based on the personal preference who are going through similar experiences or difficulties. Figure 6 illustrates the semantic social-collaborative network in the context of GRaCE-Age healthcare system with extended social relations with non-human entities. 


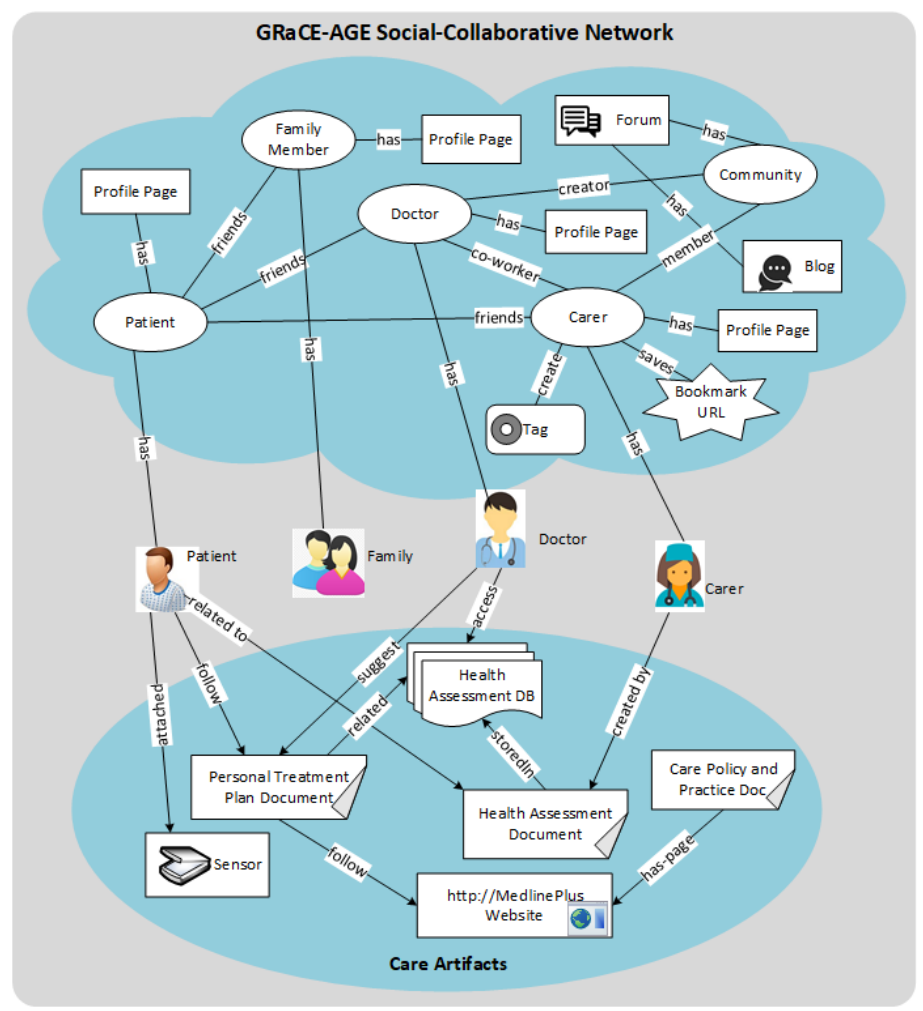

Figure 5. GRaCE-Age Social-Collaborative Healthcare Network

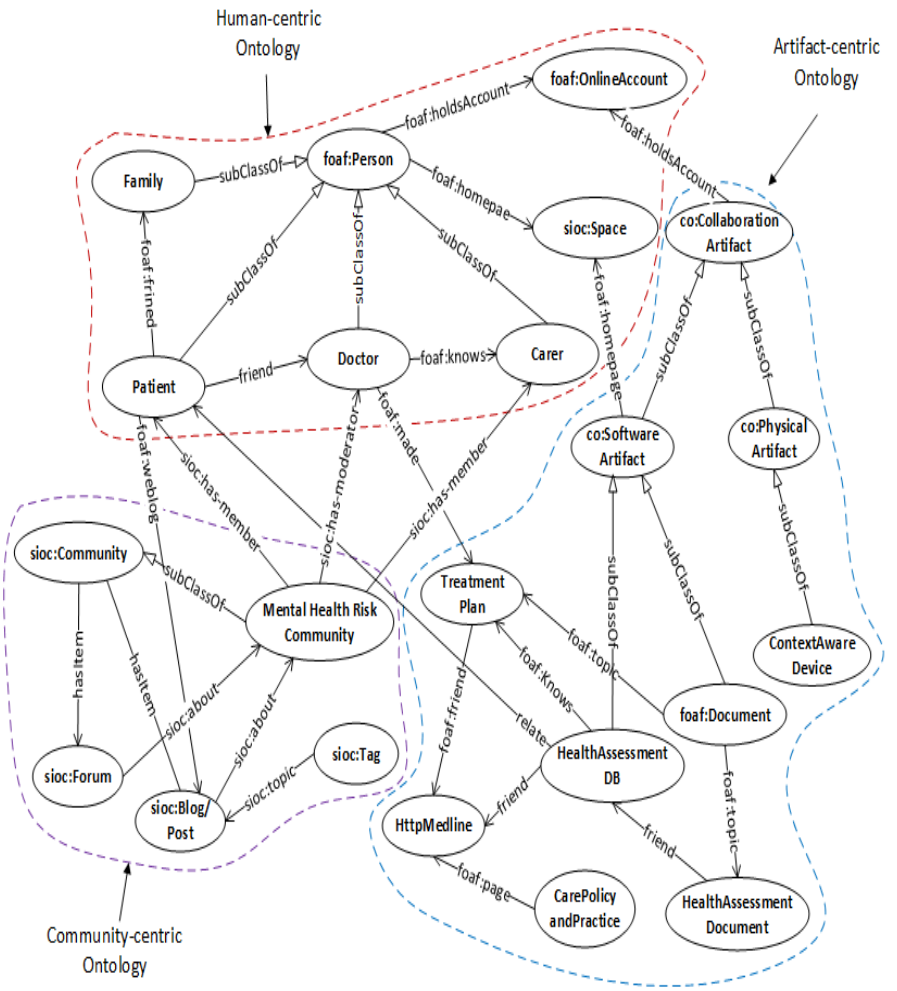

Figure 6. Care-Network Ontology 


\subsection{Scenario A - GRaCE Social Connections and Communications}

As shown in Figure 5, assume that a Patient is socially connected by friend relation with a Doctor and a Carer based on his/her care support team. This Patient is also connected with a Family member as a friend relation, and the Doctor and Carer both are connected as a $\mathrm{CO}$-worker. The recommendation system may find relevant medical information based on patient's health assessment record and suggest an additional care plans to a patient based on the inferred medical web resources (i.e., from MedlinePlus Website).

Furthermore, the Carer may save social Bookmark for the medical URL page about Care Policy and Practice, and also, he or she may create a Tag relevant to health information associated to the patient health condition. A social notification service is used as a mechanism to send an alert to reach all connected members in an emulated emergency situation.

Additionally, Patients may participate in a Discussion Forum about specific diseases or conditions, may follow particular medical treatments and procedures, and get advice on health-related topics. A discussion forum can have a moderator, such as a Doctor who creates a Care Community of practice for a particular type of health condition (i.e., mental health risk community) and allows access to others for joint discussion, post comments or reviews, etc. Building this type of care community around a specific topic is critical sources of trusted information for patients and caregivers, where they can engage with other potential members, listen to each other concerns and answer disease related questions. The social communication, interaction, event notification and relationship discussed in Scenario A will be maintained by the social networking system, which is easy to implement.

\subsection{Scenario B - Artifact-Centric Social Relations in GRaCE}

As shown in Figure 5, in this section, we illustrate in more details the complex social connections and communication between human and artifacts in the care network. These healthcare resources or artifacts are created, accessed, used in a variety of contexts, which deliver a distinctive aspect of sociality in the care network.

According to Scenario B, there may be different kinds of healthcare artifacts, including HealthAssessmentDB to store health data, HealthAssessmentDocument related to individual patient's health assessment, PersonalTreatmentPlanDocument created by doctor according to patient health condition and assessment, http://MedlinePlusWeb is a web resource that contains health information and other medical artifacts, and Care Policy and Practice documents described the various types of health information. These artifacts may socially be connected to each other as well as with the human agent by the relation of $\mathrm{friends}$, follows, storedIn, createdBy, suggests, etc. property relations. However, the limitation is that these non-human objects needs human interaction to maintain their social existence and relationship and keep track of changes in the network, which is difficult in a dynamically changing situation, such as if the links or interactions grows.

\subsection{Implementation}

In this section, we present the prototype implementation of GRaCE-Age care network ontology as shown in Figure 4 and demonstrate how to link with extended upper-level "SocialCollaborationOntology" that endorse knowledge integration within the social-collaborative care network. We built and manually merged these ontologies using Prot'eg'e-5.5.0 ontology editor (https://protege.stanford.edu/products.php\\#web-protege). Prot'eg'e is one of the most widely used ontology development tools, which is a free, open-source platform that provides a suite of tools to construct domain models and knowledge-based applications. Afterwards, we performed basic testing on ontology by launching the inference engine on RacerPro to ensure the consistency of the ontology.

\subsubsection{Interlinking Extended "SocialCollaborationOntology" with the GRaCE Network}

The purpose of interlinking ontology with a care network is to illustrate how a domain ontology reuses concepts and properties from extended upper-level ontology to describe the domain in semantic hierarchies and relationships. It will help to deduce context information from user's profile, personal medical status and shared 
objects or resources using inference rules in dedicated collaborative context. The first step is to create the mind map that describes the major concepts of GRaCE-AGE domain and then adding the properties (slots) and features (facets) to the classes to describe the structure of the care network. The classes of "CareNetworkOntology" are then constructed and linked with upper-level "SocialCollaborationOntology" and "CoreOnto" ontology with appropriate object and data properties based on the care context scenarios. The resulting ontology forming a three-different type of sub-ontologies, including Human-centric ontology, Artifact-centric ontology and Community-centric ontology separated by dash lines as shown in Figure 6.

\section{DISCUSSION AND CONCLUSION}

Web 2.0 based social-collaboration platforms enable participation, communication and cooperation in working groups based on individual's activities (Gaál et al., 2015). In this work, we illustrate the advantage and applicability of social networking approach with artifact-centric interaction, communication and information sharing in a collaborative context. In practice, we have demonstrated how social networking features utilised and impacted the way health care network of participants (human, smart devices and care artifacts) does collaboration with regards to care support. We suggest that the social context plays an essential role in describing the situation in which human and collaborative artifacts engage and communicate. In addition, we argue the benefits of ontological representation of social-collaborative network structure by extending existing social ontologies such as FOAF, SIOC, and DC that define new concepts and complex social relationship of these entities. One of the limitations of this work is that the resources in the network need human agents to maintain their social existence, relation and communication with others. Managing and keep tracking of these larger numbers of resources is a complex task for a human agent even with the help of computational tools, because of their social context and relation may change constantly as network grows. Additionally, social networks employ event notification mechanisms to notify people for relevant changes. A typical notification policy is that when an event happens at a node, the event is reported to all nodes that are directly connected to the source node. However, propagating events without overwhelming people with relevant information or alerts is another research challenge (Begel \& Deline, 2009).

Our prospective vision is that the next-generation social-collaborative system and its resources will be able to maintain their connection, communication and collaboration in an autonomous way to reduce management complexity and without human intervention.

\section{ACKNOWLEDGEMENT}

This work was supported and partially funded by the KIC EIT Health Grant for GRaCE-AGE.

\section{REFERENCES}

Abbattista, F., Calefato, F., Gendarmi, D., \& Lanubile, F. (2008). Incorporating Social Software into Distributed Agile Development Environments. In Proceedings of the 23rd IEEE/ACM International Conference on Automated Software Engineering (pp. 46-51). IEEE Press. https://doi.org/10.1109/ASEW.2008.4686310

Arnaboldi, V., Conti, M., Passarella, A., \& Dunbar, R. I. M. (2017). Online Social Networks and information diffusion: The role of ego networks. Online Social Networks and Media, 1, 44-55. https://doi.org/10.1016/j.osnem.2017.04.001

Begel, A., \& Deline, R. (2009). Codebook: Social Networking Over Code. In 31st International Conference on Software Engineering - Companion Volume. Bancouver, BC, Canada: IEEE. https://doi.org/10.1109/ICSECOMPANION.2009.5070997

Benhiba, L., Loutfi, A., Abdou, M., \& Idrissi, J. (2017). A Classification of Healthcare Social Network Analysis Applications. In BIOSTEC 2017 (pp. 978-989). Scite Press. https://doi.org/10.5220/0006168001470158

Bergstrom, A., Clark, R., Hogue, T., Iyechad, T., Miller, J., Mullen, S., ... Thurston, F. (1995). Collaboration framework: Addressing community capacity. Fargo, ND.

Berners-Lee, T., Hendler, J., \& Lassila, O. (2001). The Semantic Web. Scientific American, 284(5), 34-43. Retrieved from https://www.jstor.org/stable/26059207 
Buckingham, C. D., Adams, A., Vail, L., Kumar, A., Ahmed, A., Whelan, A., \& Karasouli, E. (2015). Integrating service user and practitioner expertise within a web-based system for collaborative mental-health risk and safety management. Patient Education and Counseling, 98(10), 1189-1196. https://doi.org/10.1016/j.pec.2015.08.018

Chen, R. C., Hendry, \& Huang, C.-Y. (2016). A Domain Ontology in Social Networks for Identifying User Interest for Personalized Recommendations. Journal of Universal Computer Science, 22(3), 319-339.

Christopoulou, S. C., Kotsilieris, T., Anagnostopoulos, I., Anagnostopoulos, C. N., \& Mylonas, P. (2017). vhMentor: An Ontology Supported Mobile Agent System for Pervasive Health Care Monitoring. Advances in Experimental Medicine and Biology, 989, 57-65. https://doi.org/10.1007/978-3-319-57348-9_5

Dey, N., Ashour, A. S., Shi, F., Fong, S. J., Manuel, J., \& Tavares, R. S. (2018). Medical cyber-physical systems: A survey. Journal of Medical Systems, 42(74). https://doi.org/10.1007/s10916-018-0921-x

Engeström, J. (2005). Object-centered sociality. Retrieved from http://aula.org/people/jyri/presentations/reboot7-jyri.pdf

Gaál, Z., Szabó, L., Obermayer-Kovács, N., \& Csepregi, A. (2015). Exploring the Role of Social Media in Knowledge Sharing. The Electronic Journal of Knowledge Management, 13(3), 185-197.

Ghafoor, F., \& Niazi, M. A. (2016). Using social network analysis of human aspects for online social network software: a design methodology. Complex Adaptive Systems Modeling, 4(1), 14. https://doi.org/10.1186/s40294-016-0024-9

Hussain, N., Wang, H. H., \& Buckingham, C. D. (2018). Policy Based Generic Autonomic Adapter for a Context-Aware Social-Collaborative System. In International Conference on Intelligent Systems and Computer Vision (ISCV) (pp. 1-9). Fes, Morocco: IEEE. Retrieved from https://ieeexplore.ieee.org/document/8354044/

Kaldoudi, E., Dovrolis, N., Giordano, D., \& Dietze, S. (2011). Educational Resources as Social Objects in Semantic Social Networks. In Proceedings of the Linked Learning (Vol. 717). Greece: CiteSeerX. https://doi.org/10.1.1.369.6525

Koch, M. (2008). CSCW and Enterprise 2.0 - towards an integrated perspective. In 21th Bled eConference eCollaboration: Overcoming Boundaries Through Multi-Channel Interaction (pp. 1-15). Bled, Slovenia. https://doi.org/10.1111/j.1365-2656.2008.01372.x

Lapointe, L., Ramaprasad, J., \& Vedel, I. (2014). Creating health awareness: A social media enabled collaboration. Health and Technology, 4(1), 43-57. https://doi.org/10.1007/s12553-013-0068-1

Lima, C., Antunes, M., Gomes, D., Aguiar, R., \& Mota, T. (2014). A Context-Aware Framework for Collaborative Activities in Pervasive Communities. International Journal of Distributed Systems and Technologies, 5(2), 31-43. https://doi.org/10.4018/ijdst.2014040103

Liptchinsky, V., Khazankin, R., Schulte, S., Satzger, B., Truong, H. L., \& Dustdar, S. (2014). On modeling context-aware social collaboration processes. Information Systems, 43, 66-82. https://doi.org/10.1016/j.is.2013.05.007

Lohmann, S., Dietzold, S., Heim, P., \& Heino, N. (2009). A web platform for social requirements engineering. Software Engineering, 309-315.

Marie, N., \& Gandon, F. (2011). Social objects description and recommendation in multidimensional social networks: OCSO Ontology and semantic spreading activation. Proceedings - 2011 IEEE International Conference on Privacy, Security, Risk and Trust and IEEE International Conference on Social Computing, PASSAT/SocialCom 2011, 1415-1420. https://doi.org/10.1109/PASSAT/SocialCom.2011.242

Nansen, B., van Ryn, L., Vetere, F., Robertson, T., Brereton, M., \& Douish, P. (2014). An internet of social things. Proceedings of the 26th Australian Computer-Human Interaction Conference on Designing Futures the Future of Design - OzCHI '14, 87-96. https://doi.org/10.1145/2686612.2686624

Obeid, C., Lahoud, I., El Khoury, H., \& Champin, P.-A. (2018). Ontology-based Recommender System in Higher Education. Companion of the The Web Conference 2018 on The Web Conference 2018, 2, 1031-1034. https://doi.org/10.1145/3184558.3191533

Oellinger, T., \& Wennerberg, Pinar Oezden. (2006). Ontology Based Modeling and Visualization of Social Networks for the Web. In GI Jahrestagung, 7(2), 489-497.

Samuel, A. (2015). Collaborating Online Is Sometimes Better than Face-to-Face. Retrieved November 3, 2018, from https://hbr.org/2015/04/collaborating-online-is-sometimes-better-than-face-to-face

Soto, M. V., Balls-Berry, J. E., Bishop, S. G., Aase, L. A., Timimi, F. K., Montori, V. M., \& Patten, C. A. (2016). Use of Web 2.0 Social Media Platforms to Promote Community-Engaged Research Dialogs: A Preliminary Program Evaluation. JMIR Research Protocols, 5(3). https://doi.org/10.2196/resprot.4808

Stan, J., \& Villarceaux, C. De. (2009). An overview and research vision of the use of Semantic Technologies for User Modeling and Collaborative Tagging. https://doi.org/10.1.1.299.8477

Xiao, Y. (2005). Artifacts and collaborative work in healthcare: Methodological, theoretical, and technological implications of the tangible. Journal of Biomedical Informatics, 38(1), 26-33. https://doi.org/10.1016/j.jbi.2004.11.004

Zaffar, F. O., \& Ghazawneh, A. (2012). Knowledge Sharing and Collaboration Through Social Media - The Case of IBM. In Proceedings of the 7th Mediterranean Conference on Information Systems, MCIS (Vol. 170, pp. 1-11). Association for Information Systems. 